\title{
Enhancing open-set face recognition by closing it with Cluster-Inferred Gallery Augmentation
}

\author{
Floris De Feyter ${ }^{[0000-0003-2690-0181]}$, Kristof Van Beeck ${ }^{[0000-0002-3667-7406]}$, \\ and Toon Goedemé $[0000-0002-7477-8961]$ \\ KU Leuven-EAVISE \\ Jan De Nayerlaan 5, 2860 Sint-Katelijne-Waver, Belgium \\ https://iiw.kuleuven.be/onderzoek/eavise \\ floris.defeyter@kuleuven. be
}

\begin{abstract}
In open-set face recognition-as opposed to closed-set face recognition - it is possible that the identity of a given query is not present in the gallery set. In that case, the identity of the query can only be correctly classified as "unknown" when the similarity with the gallery faces is below a threshold that was determined a priori. However, in many use-cases, the set of queries contains multiple instances of the same identity, whether or not this identity is represented in the gallery. Thus, the set of query faces lends itself to identity clustering that could yield representative instances for unknown identities. By augmenting the gallery with these instances, we can make an open-set face recognition problem more closed. In this paper, we show that this method of Cluster-Inferred Gallery Augmentation (CIGA) does indeed improve the quality of openset face recognition. We evaluate the addition of CIGA for both a private dataset of images taken in a school context and the public LFW dataset, showing a significant improvement in both cases. Moreover, an implementation of the suggested approach along with our experiments are made publicly available on https://gitlab.com/florisdf/acpr2019.
\end{abstract}

Keywords: Open-set face recognition - Face clustering · Gallery augmentation.

\section{Introduction}

Facial recognition can be categorized as face verification and face identification [23]. Both paradigms work with a set of known faces (the gallery), and an unknown face (the query or probe) that needs to be identified. In face verification, the query is compared in a one-to-one fashion to the gallery items. Each of these comparisons yields either a match (same identity) or a mismatch (different identity). In face identification, the query is compared to the whole gallery at once in a one-to-many fashion, yielding the identity of the best matching gallery item. For both face recognition categories, it is possible that the identity of the query face is not present in the gallery. This is referred to as open-set recognition. 

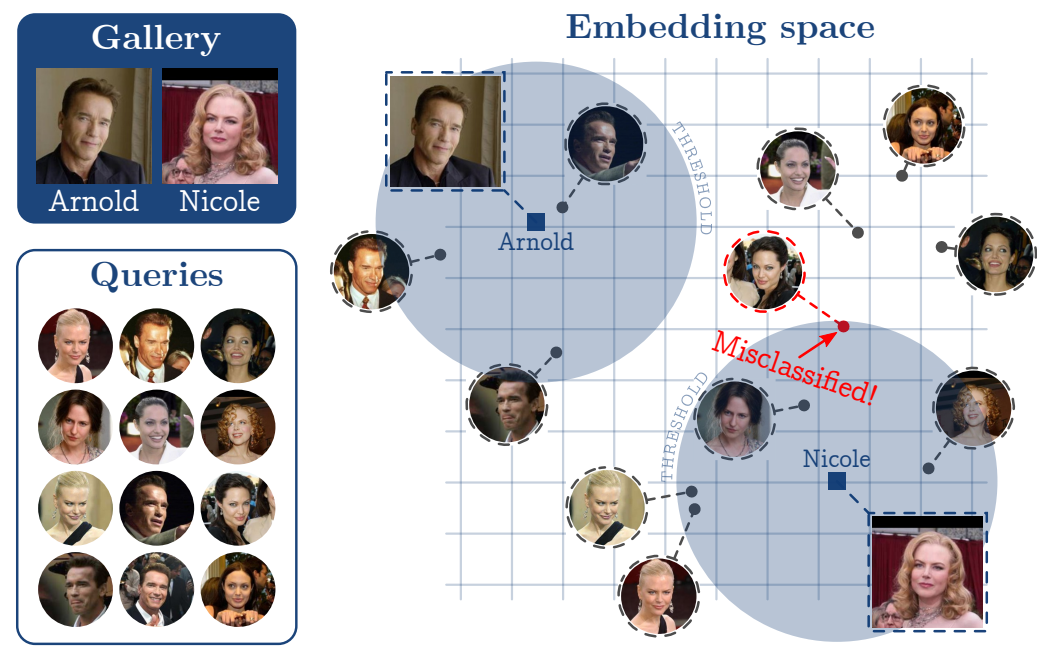

Fig. 1: Simply thresholding a similarity measure might not suffice to avoid problems with open-set recognition. Here, Angelina Jolie is unknown and one of her embeddings is misclassified as "Nicole" because it falls within the threshold limit. The fact that the embedding is close to other embeddings of Angelina Jolie, is ignored.

When all query identities are present in the gallery, the recognition is referred to as closed-set.

\subsection{Closing Open-Set Recognition}

The problem with open-set face recognition is that when a given query does not appear in the gallery, the system will certainly misclassify the query. This misclassification can be prevented, however, by setting a proper threshold on the similarity score of the query face and the gallery items. Whenever the similarity score between query and gallery is below such a threshold, the system could report that the query has an unknown identity. Still, with this approach, much emphasis is put on the threshold and any query of an unknown identity with a similarity score slightly above the threshold, will certainly be misclassified. Figure 1 illustrates this problem. Two identities are present in the gallery: "Arnold" and "Nicole". In the query set, however, a third unknown identity is present (Angelina Jolie). One of these unknown queries has a face embedding that is just close enough to the gallery embedding of "Nicole" to fall within the threshold limit and get misclassified as "Nicole".

At the same time, many identities will occur more than once in the set of all queries. For example, when searching for a person through security camera footage, most people will be visible in multiple frames and thus appear more than once as a query. Therefore, the set of queries has a valuable structure that 
would be ignored when we simply focus on setting a good threshold to avoid misclassifications. More specifically, one can try to exploit the structure of the query set to infer reference images of identities that are not present in the gallery set. These references of unknown identities can then be added to the gallery set. As such, one makes the open-set recognition problem more closed by adding identities to the gallery that previously were not present but that do appear in the query set. This will alleviate the stress on setting a good threshold, since a false positive matching score that was slightly above the threshold for one of the known gallery items, can now have a much higher true positive matching score with one of the unknown identities in the augmented gallery. Hence, the queries that do match with the known identities will be more reliable, leading to a more precise identification system. This solution is shown in Figure 2. The embeddings of the query set are analyzed and a third identity is discovered, labeled "id3". A representative embedding is chosen and added to the gallery. The query that was misclassified in Fig. 1, has a better match with this new embedding and is therefore not classified as "Nicole", but as "id3".
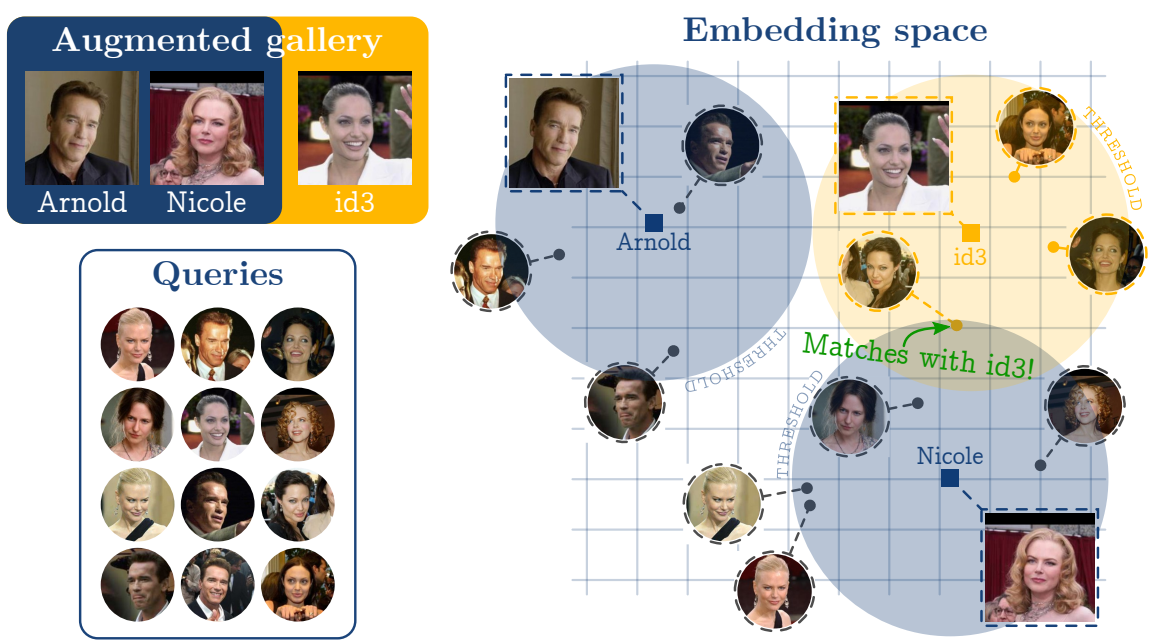

Fig. 2: By inspecting the structure of the query embeddings, we can augment the gallery with identities that were previously unknown. That way, a previously false positive match can now have a much better true positive match with the new gallery item.

We propose a proof-of-concept that is based on an initial step of clustering face embeddings to augment the original gallery with extra (unknown) identities. Section 3 discusses this approach - which we coin Cluster-Inferred Gallery Augmentation (CIGA) - in more detail. 


\subsection{Use-Case: Individualized School Photo Albums}

As a concrete example, we frame our proposed approach in the use-case of composing individualized school photo albums. After a year of photographing the school activities and memorable moments, teachers have created a collection of photos that can be very valuable to many parents. However, most parents are mainly interested in pictures of their own child. A facial recognition system could be used to identify the faces in each picture, based on a reference image that parents provide. These references form the gallery of the identification system, since we know the identity of each reference image. The faces in the pictures that were taken throughout the year correspond to the query set, i.e. we want to identify each of these faces by comparing them to the reference images. This use-case is indeed an open-set recognition problem as we assume that there will be children in the query set of whom the parents did not provide a reference image.

In the evaluation of our proposed approach, we use a private real-world dataset - referred to as the Jade dataset - that was created for the development of such an album-creating system. The Jade dataset contains 1629 labeled images of 29 unique identities. It contains a wide variety of image types: close-up portraits, wider portraits, group photos etc. Figure 3 shows multiple randomly selected samples of the Jade dataset. The color of the bounding boxes corresponds to the identity of the face. It is clear that the images are unconstrained in terms of pose, facial expression, face angle, blur, resolution and lighting.

\section{Related Work}

Much research has been done on open-set recognition, mostly in the general case of open-set image classification $[1-3,12,13,16]$. Scheirer et al. [16] were the first to formally define open-set recognition as a constrained minimization problem. They propose a 1-vs-set SVM that has a second plane in the feature space which delimits the reasonable support of known classes. Many other papers further built upon this idea $[1,10,17,18]$. Apart from the 1-vs-set-based approaches, an algorithm based on deep neural networks has also been proposed [2]. Bendale and Boult present a methodology that adds an extra layer - called OpenMaxto the network. The additional layer estimates the probability that an input belongs to an unknown class.

The more specific case of open-set face recognition is often considered and evaluated $[8,20]$, but only rarely algorithms are specifically targeted at dealing with open-set situations [6]. Instead, most works on face recognition focus on creating algorithms that transform faces into robust embeddings $[4,14,19,21$, $22]$. They assume that a robust embedding combined with a proper threshold should prevent problems with open-set recognition.

The previous works ignore that in many practical situations of open-set recognition, the query set contains multiple examples of the same unknown classes. For example, in our use-case of individualized school album creation described 

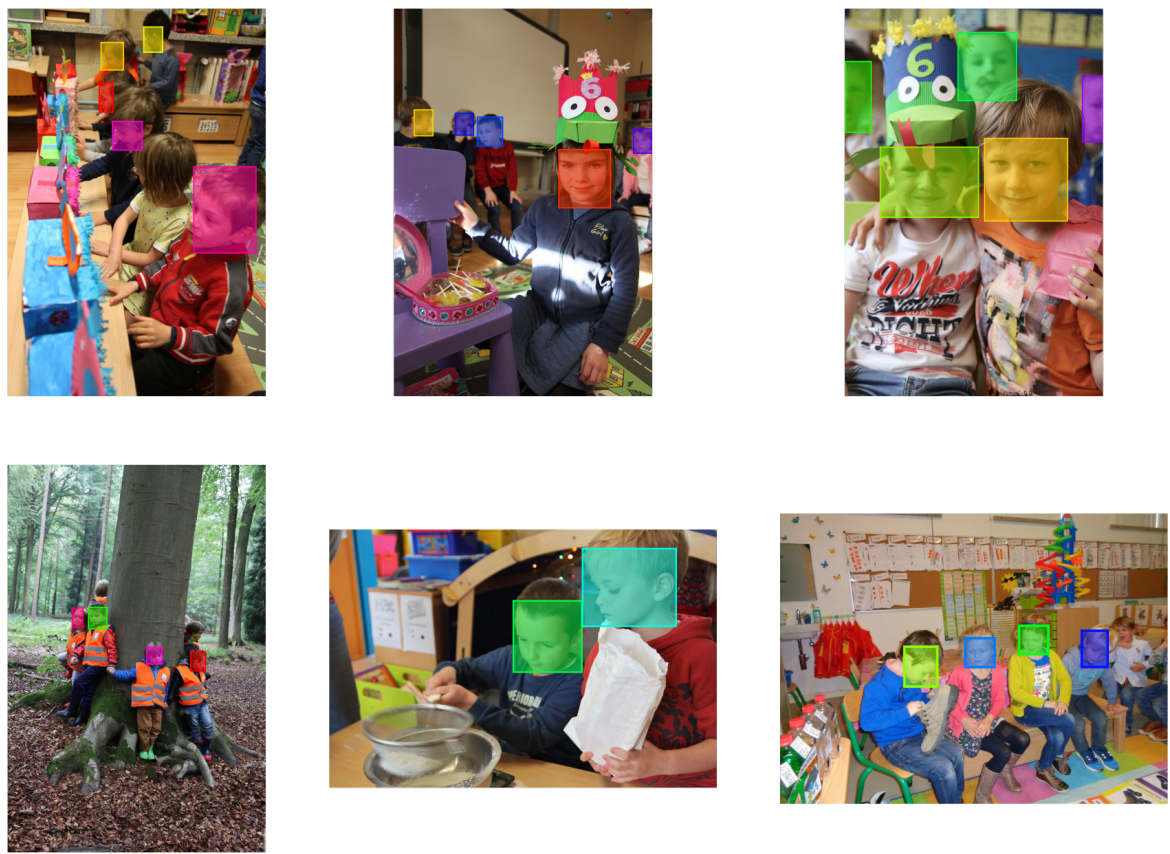

Fig. 3: Random samples from the Jade dataset of labeled school images. The color of the bounding boxes represents the identity of the child. 
in Sec. 1.2, an unknown child that is in the same class as a known child, will probably appear multiple times in the series of images. Our approach attempts to exploit this query structure to discover unknown identities such that the recognition of the identities of interest (i.e. the known identities) will improve.

\section{Cluster-Inferred Gallery Augmentation}

As previously motivated, discovering the structure of the query set might improve the precision of a facial recognition system that is used in an open-set context. More specifically, we argue that one can infer representative instances of identities that are not present in the gallery set but that are present in the query set. We propose an approach called Cluster-Inferred Gallery Augmentation (CIGA). It consists of the following steps:

1. Add the descriptors from the query set and the gallery (i.e. the known identities) into one large set of descriptors and cluster this set of descriptors;

2. Discard the clusters where items from the original gallery ended up;

3. For each remaining cluster, find the medoid;

4. Add the cluster medoids as new identities to the gallery;

5. Discard newly added gallery items that lie unusually close to another gallery item.

Note that we made the CIGA algorithm publicly available via this repository: https://gitlab.com/florisdf/acpr2019. The README file explains how you can use the CIGA algorithm yourself. The following sections explain the algorithm in more detail.

\subsection{Clustering}

We propose to exploit the structure of the query set by clustering the face embeddings. It is, however, unfeasible to make a realistic a priori estimation of the amount of separate identities that are present in the dataset. Therefore, a clustering algorithm like K-means clustering [7] is inapplicable for CIGA as it takes the number of clusters as an argument. In our proof-of-concept, we use the Density-Based Spatial Clustering of Applications with Noise (DBSCAN) algorithm [5] to discover the query structure. This algorithm defines clusters as areas of high density separated by areas of low density. The algorithm has two parameters: $\varepsilon$ and min_samples. When at least min_samples samples are within a distance $\varepsilon$ of a sample (including the sample itself), that sample is considered a core sample. A sample that is in the neighbourhood of a core sample can either be a core sample itself, or a non-core sample, which means that there are less than min_samples samples within a distance $\varepsilon$ around that sample. A cluster contains at least one core sample, and hence at least min_samples samples. Samples that are not in the neighbourhood of a core sample, do not belong to a cluster and are considered outliers. Figure 4 shows the DBSCAN algorithm applied on a small set of data points. 


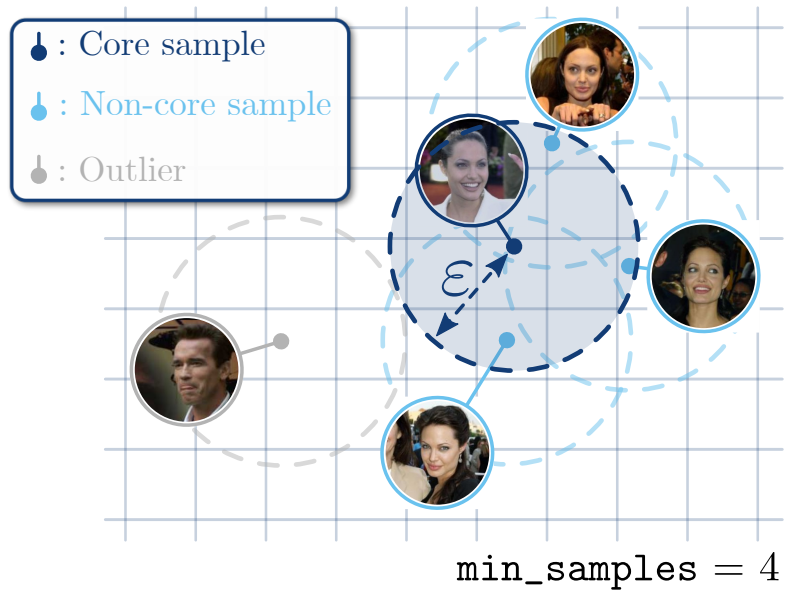

Fig. 4: The DBSCAN algorithm applied on a set of 5 points. The parameter min_samples is set to 4 and the distance $\varepsilon$ is chosen as shown in the figure. There is one core sample (dark blue), three non-core samples (light blue) and one outlier (grey). As such, the clustering results in one cluster (the blue points) and one outlier.

\subsection{Selecting References for Unknown Identities}

After clustering the query and gallery faces, we want to select references for unknown identities to add to the gallery. However, we must avoid augmenting the gallery with an already known identity, since, in that case, query faces of that known identity might later get classified as unknown. Therefore, we look for the clusters where the original gallery faces end up. These clusters are ignored for finding new gallery items. For the remaining clusters, we choose to add the medoid embedding to the gallery as a new identity, i.e. the embedding that has, on average, the smallest distance to the other embeddings in the same cluster. This process is illustrated in Figure 5. The clustering resulted in 3 clusters. However, two of them contain an item from the original gallery. These two clusters are therefore ignored. For the remaining cluster, we search the medoid and add this as a reference of a new, unknown, identity to the gallery. Hence, we obtain the augmented gallery.

\subsection{Discarding Similar Gallery Items}

The final step involves calculating the mean difference between all the embeddings in the augmented gallery set and removing the descriptors that lie closer than $k$ standard deviations from the mean difference. This is, again, to avoid that an identity that was already in the gallery would have a second representation that is marked as unknown. In our experiments, we chose a fixed $k=3$. Further experiments are needed to determine the best value of this parameter and to evaluate its influence on the performance of CIGA. 


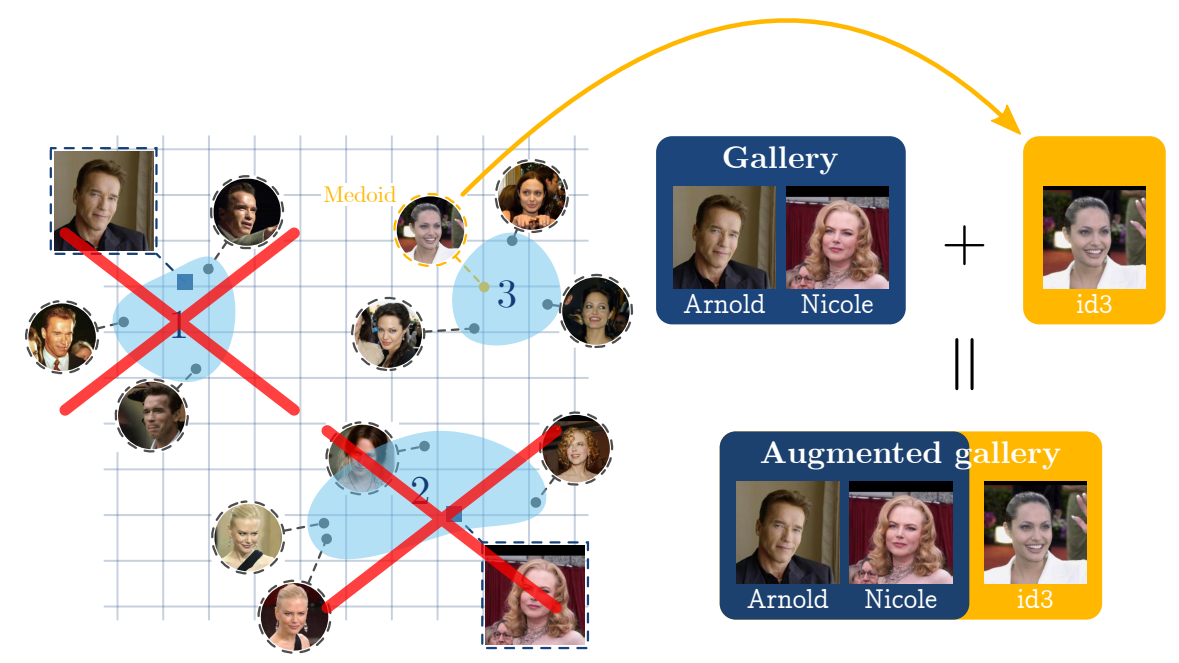

Fig. 5: After clustering the query and gallery embeddings, we ignore clusters with gallery items. For the remaining cluster, the medoid is used to add as a reference of a new identity to the gallery.

\section{Experiments and Results}

In the following sections, we evaluate our proposed method that involves exploiting the structure of the query set to improve the classification result of an open-set recognition problem. In Sec. 4.1, we perform a grid search to find the optimal parameters for the DBSCAN clustering algorithm that is used by CIGA. Next, in Sec. 4.2 we evaluate CIGA on both the Jade dataset and a subset of the LFW dataset. The experiments from Sec. 4.2 can be reproduced from the code on our repository: https://gitlab.com/florisdf/acpr2019. The README file contains further details on how to reproduce the results.

\subsection{DBSCAN Hyperparameters}

The values to choose for the parameters $\varepsilon$ and min_samples of the DBSCAN clustering algorithm, depend on the density of the descriptors to cluster. We performed a grid search to discover the hyperparameters that yield truthful clusters. We used two metrics for evaluating the clustering results: the Adjusted Rand Index (ARI) and the number of clusters found. The ARI is a metric to determine the similarity of two clusterings, with chance normalization [9]. It yields a result between -1 and 1 where 1 means that the two clusterings are identical. The ground truth clusters are obtained by simply grouping the labeled faces per identity.

Our experiments are done on the Jade dataset, assuming that - when using the same facial feature extractor - the hyperparameters would generalize to other datasets as well. This assumption will be confirmed in Section 4.2. Figure 6 (a) 
shows the ARI between the clusters arising from the DBSCAN clustering and the ground truth clusters for multiple combinations of $\varepsilon$ and min_samples. The darker the color, the closer the ARI is to 1 and thus the better the DBSCAN clustering. While the highest ARI scores are obtained in the leftmost column of the heat map, setting min_samples to 1 would not be a good choice as is apparent from Figure $6(\mathrm{~b})$, which shows that the number of clusters becomes very high in that case. This makes sense, because min_samples $=1$ means that every sample is a cluster by itself. Ideally, the number of clusters would be equal to $30(=29+1$ for outliers) corresponding to the 29 identities present in the Jade dataset. The color map in Figure 6 (b) is centered around 30, such that the darkest areas are those with the correct amount of clusters. When comparing Figs. 6 (a) and (b), a reasonable choice for the parameters would be $\varepsilon=0.42$ and min_samples $=5$.

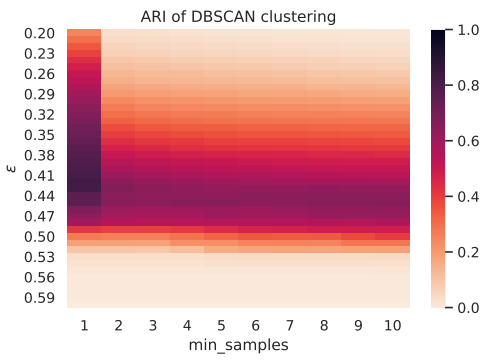

(a) ARI vs. DBSCAN hyperparameters

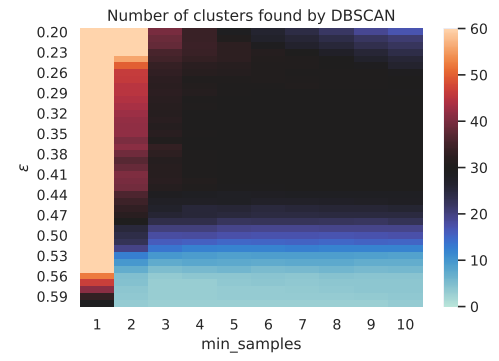

(b) Number of clusters vs. DBSCAN hyperparameters

Fig. 6: Two types of grid search to find the optimal parameters for the DBSCAN algorithm. (a) ARI between the DBSCAN clustering and the ground truth clusters for multiple combinations of $\varepsilon$ and min samples; darker is better. (b) Number of clusters according to the DBSCAN clustering for multiple combinations of $\varepsilon$ and min samples; darker is better.

\subsection{CIGA in an Open-Set Face Recognition Pipeline}

We evaluate the effect of adding CIGA for two different datasets: our private Jade dataset and a subset of the publicly available LFW [8] dataset. In both cases, we use the MTCNN face detector [24], a facial feature extractor from the Dlib library [11] and the DBSCAN clustering algorithm [5] from the Scikit-learn library [15]. For each dataset, we take the following steps after detecting the faces and extracting the face embeddings:

1. Compose a random gallery with $N_{\text {gal }}$ items;

2. Apply CIGA, adding $N_{C I G A}$ extra items to the gallery; 
3. Classify the query items into the $N_{g a l}+N_{C I G A}$ classes defined by the new gallery using Euclidean distance as a similarity measure;

4. Calculate the average precision (AP) score of the micro-averaged PR-curve for the classification of the identities that were initially in the gallery, i.e. the known identities.

This experiment is repeated multiple times for different values of $N_{\text {gal }}$. For each of these values, we repeat the experiment 30 times, each time randomly selecting a certain combination of $N_{\text {gal }}$ initial gallery items. For the Jade dataset, $N_{\text {gal }}$ is varied between $3 \ldots 29$. For the LFW dataset, $N_{\text {gal }}$ is varied between $3 \ldots 150$. It is important to note here that we only used a subset of the LFW dataset, i.e. we used the 150 identities with the most images. We do this because many identities in the LFW dataset have only a single image. Of course, these identities are of no interest to the current experiment, as we want to check if exploiting the query structure improves the classification result. Therefore, the query set must contain multiple instances of the same identity. To reproduce our results, please visit https://gitlab.com/florisdf/acpr2019.

Figs. 7 and 8 show the results for the Jade and the LFW dataset, respectively. The solid lines indicate the mean of the AP score (mAP) for the 30 experiments performed with the corresponding $N_{\text {gal }}$. The colored bands around the solid lines show the standard deviation between the APs of those 30 experiments. We observe that in both cases, using CIGA systematically improves the classification result when the gallery contains few items. Table 1 shows the results for the extreme case when only 3 identities were initially present in the gallery, i.e. the leftmost value on the horizontal axis of Figs. 7 and 8. We see that CIGA indeed improves the mAP in both cases. Furthermore, the experiments show a smaller standard deviation when CIGA is used, indicating that CIGA is also a reliable improvement. This decrease in standard deviation is more than a factor of 10 for the LFW dataset. The fact that we see an improvement for both datasets, motivates the assumption on the generalizability of the DBSCAN hyperparameters found in Sec. 4.1. Lastly, we observe that the mAP clearly reduces when $N_{\text {gal }}$ increases in the case of CIGA. This is because, when more identities are initially known, the relative amount of unknown identities decreases. Hence, the improvement that CIGA offers will be less pronounced.

Table 1: Results on the Jade dataset and the LFW dataset when only 3 identities were initially present in the gallery.

\begin{tabular}{lll}
\hline Dataset & CIGA $(\mathrm{mAP})$ & No CIGA $(\mathrm{mAP})$ \\
\hline \hline Jade & $(59.12 \pm 11.65) \%$ & $(44.61 \pm 13.39) \%$ \\
LFW & $(99.74 \pm 0.46) \%$ & $(94.65 \pm 6.03) \%$ \\
\hline
\end{tabular}




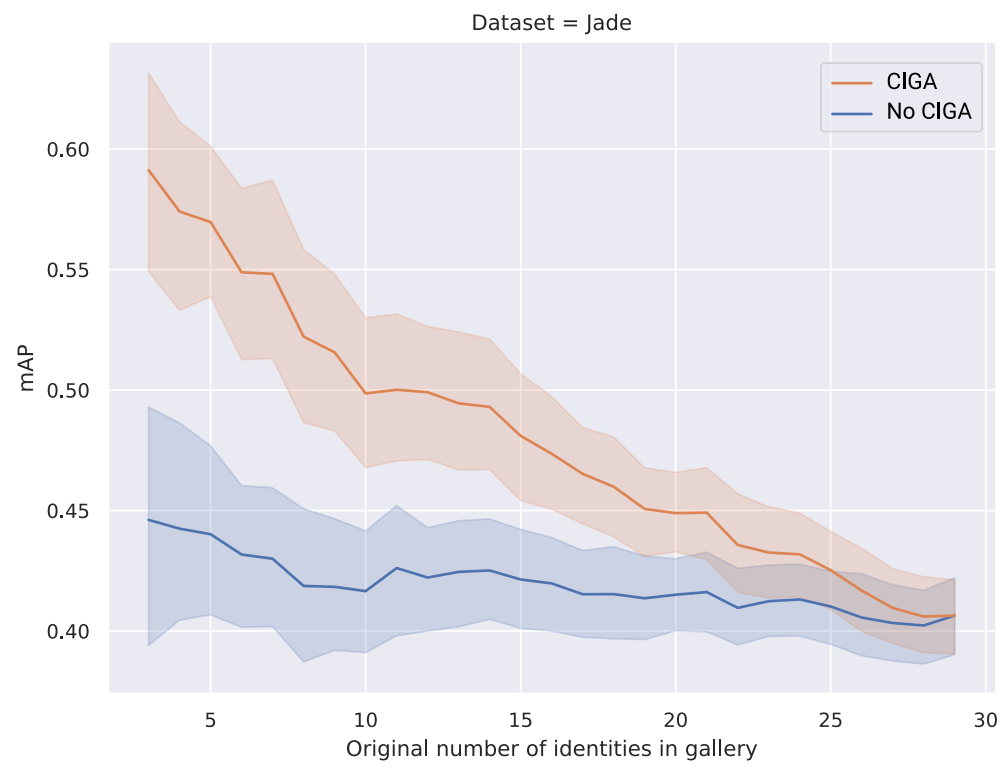

Fig. 7: Evaluation of CIGA on the Jade dataset.

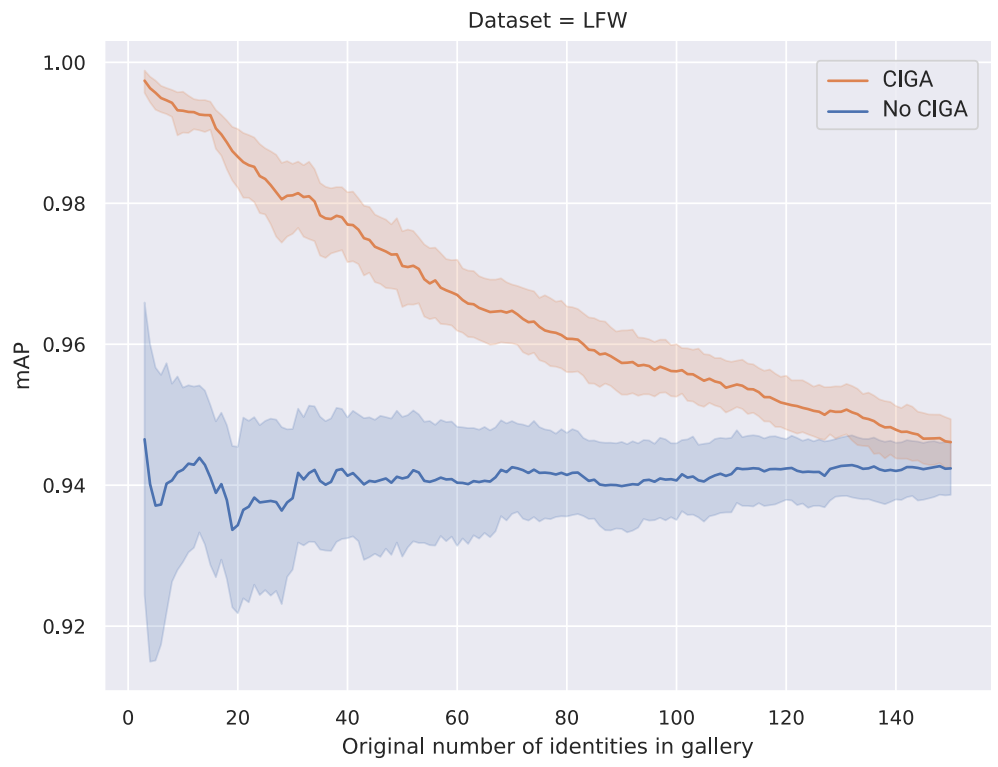

Fig. 8: Evaluation of CIGA on the LFW dataset. 


\section{Conclusion}

In this paper, we motivate that in many practical open-set face recognition applications a query might contain a valuable structure that is often ignored. We proposed a proof-of-concept that consists of a Cluster-Inferred Gallery Augmentation step and evaluated it on a private real-world dataset and a subset of the public LFW dataset. As such, we showed that exploiting the query structure can indeed significantly improve the performance of an open-set face recognition problem. Our implementation of the algorithm and the experiments can be found on https://gitlab.com/florisdf/acpr2019.

\section{References}

1. Bendale, A., Boult, T.: Towards Open World Recognition. In: The IEEE Conference on Computer Vision and Pattern Recognition (CVPR). pp. 1893-1902 (2015)

2. Bendale, A., Boult, T.E.: Towards Open Set Deep Networks. In: Proceedings of the IEEE conference on computer vision and pattern recognition. pp. $1563-1572$ (2016)

3. Busto, P.P., Iqbal, A., Gall, J.: Open set domain adaptation for image and action recognition. IEEE Transactions on Pattern Analysis and Machine Intelligence (2018)

4. Deng, J., Guo, J., Niannan, X., Zafeiriou, S.: Arcface: Additive angular margin loss for deep face recognition. In: CVPR (2019)

5. Ester, M., Kriegel, H.P., Sander, J., Xu, X., et al.: A density-based algorithm for discovering clusters in large spatial databases with noise. In: Kdd. vol. 96, pp. 226-231 (1996)

6. Günther, M., Cruz, S., Rudd, E.M., Boult, T.E.: Toward Open-Set Face Recognition. In: Proceedings of the IEEE Conference on Computer Vision and Pattern Recognition Workshops. pp. 71-80 (2017)

7. Hartigan, J.A., Wong, M.A.: Algorithm as 136: A k-means clustering algorithm. Journal of the Royal Statistical Society. Series C (Applied Statistics) 28(1), 100$108(1979)$

8. Huang, G.B., Mattar, M., Berg, T., Learned-Miller, E.: Labeled faces in the wild: A database forstudying face recognition in unconstrained environments. In: Workshop on faces in'Real-Life'Images: detection, alignment, and recognition (2008)

9. Hubert, L., Arabie, P.: Comparing partitions. Journal of classification 2(1), 193$218(1985)$

10. Jain, L.P., Scheirer, W.J., Boult, T.E.: Multi-class open set recognition using probability of inclusion. In: Lecture Notes in Computer Science (including subseries Lecture Notes in Artificial Intelligence and Lecture Notes in Bioinformatics). pp. 393-409 (2014). https://doi.org/10.1007/978-3-319-10578-9_26

11. King, D.E.: Dlib-ml: A machine learning toolkit. Journal of Machine Learning Research 10(Jul), 1755-1758 (2009)

12. Malalur, P., Jaakkola, T.: Alignment Based Matching Networks for One-Shot Classification and Open-Set Recognition. arXiv preprint arXiv:1903.06538 (2019)

13. Meyer, B.J., Drummond, T.: The importance of metric learning for robotic vision: Open set recognition and active learning. arXiv preprint arXiv:1902.10363 (2019)

14. Parkhi, O.M., Vedaldi, A., Zisserman, A., et al.: Deep face recognition. In: bmvc. vol. 1, p. 6 (2015) 
15. Pedregosa, F., Varoquaux, G., Gramfort, A., Michel, V., Thirion, B., Grisel, O., Blondel, M., Prettenhofer, P., Weiss, R., Dubourg, V., Vanderplas, J., Passos, A., Cournapeau, D., Brucher, M., Perrot, M., Duchesnay, E.: Scikit-learn: Machine learning in Python. Journal of Machine Learning Research 12, 2825-2830 (2011)

16. Scheirer, W.J., De Rezende Rocha, A., Sapkota, A., Boult, T.E.: Toward open set recognition. IEEE Transactions on Pattern Analysis and Machine Intelligence 35(7), 1757-1772 (2013). https://doi.org/10.1109/TPAMI.2012.256

17. Scheirer, W.J., Jain, L.P., Boult, T.E.: Probability models for open set recognition. IEEE Transactions on Pattern Analysis and Machine Intelligence 36(11), 23172324 (2014). https://doi.org/10.1109/TPAMI.2014.2321392

18. Scherreik, M.D., Rigling, B.D.: Open set recognition for automatic target classification with rejection. IEEE Transactions on Aerospace and Electronic Systems 52(2), 632-642 (2016). https://doi.org/10.1109/TAES.2015.150027

19. Schroff, F., Kalenichenko, D., Philbin, J.: Facenet: A unified embedding for face recognition and clustering. In: Proceedings of the IEEE conference on computer vision and pattern recognition. pp. 815-823 (2015)

20. Sun, Y., Liang, D., Wang, X., Tang, X.: Deepid3: Face recognition with very deep neural networks. arXiv preprint arXiv:1502.00873 (2015)

21. Taigman, Y., Yang, M., Ranzato, M., Wolf, L.: Deepface: Closing the gap to humanlevel performance in face verification. In: Proceedings of the IEEE conference on computer vision and pattern recognition. pp. 1701-1708 (2014)

22. Wang, H., Wang, Y., Zhou, Z., Ji, X., Gong, D., Zhou, J., Li, Z., Liu, W.: Cosface: Large margin cosine loss for deep face recognition. In: Proceedings of the IEEE Conference on Computer Vision and Pattern Recognition. pp. 5265-5274 (2018)

23. Wang, M., Deng, W.: Deep face recognition: a survey. arXiv preprint arXiv:1804.06655 (2018)

24. Zhang, K., Zhang, Z., Li, Z., Qiao, Y.: Joint face detection and alignment using multitask cascaded convolutional networks. IEEE Signal Processing Letters 23(10), 1499-1503 (2016) 